\title{
Educational outreach visits to improve venous thromboembolism prevention in hospitalised medical patients: a prospective before-and-after intervention study
}

Jed Duff ${ }^{1,2,3^{*}}$, Abdullah Omari ${ }^{1}$, Sandy Middleton ${ }^{2,4}$, Elizabeth Mclnnes ${ }^{2,4}$ and Kim Walker ${ }^{1,3}$

\begin{abstract}
Background: Despite the availability of evidence-based guidelines on venous thromboembolism (VTE) prevention clinical audit and research reveals that hospitalised medical patients frequently receive suboptimal prophylaxis. The aim of this study was to evaluate the acceptability, utility and clinical impact of an educational outreach visit (EOV) on the provision of VTE prophylaxis to hospitalised medical patients in a 270 bed acute care private hospital in metropolitan Australia.
\end{abstract}

Methods: The study used an uncontrolled before-and-after design with accompanying process evaluation. The acceptability of the intervention to participants was measured with a post intervention survey; descriptive data on resource use was collected as a measure of utility; and clinical impact (prophylaxis rate) was assessed by pre and post intervention clinical audits. Doctors who admit $>40$ medical patients each year were targeted to receive the intervention which consisted of a one-to-one educational visit on VTE prevention from a trained peer facilitator. The EOV protocol was designed by a multidisciplinary group of healthcare professionals using social marketing theory.

Results: Nineteen (73\%) of 26 eligible participants received an EOV. The majority $(n=16,85 \%)$ felt the EOV was effective or extremely effective at increasing their knowledge about VTE prophylaxis and 15 (78\%) gave a verbal commitment to provide evidence-based prophylaxis. The average length of each visit was 15 minutes (IQ range 15 to 20) and the average time spent arranging and conducting each visit was 92 minutes (IQ range 78 to 129). There was a significant improvement in the proportion of medical patients receiving appropriate pharmacological VTE prophylaxis following the intervention (54\% to 70\%, 16\% improvement, 95\% Cl 5 to 26, p=0.004).

Conclusions: EOV is effective at improving doctors' provision of pharmacological VTE prophylaxis to hospitalised medical patients. It was also found to be an acceptable implementation strategy by the majority of participants; however, it was resource intensive requiring on average 92 minutes per visit.

Keywords: Educational outreach visit, Implementation science, Venous thromboembolism prevention

\footnotetext{
* Correspondence: jduff@stvincents.com.au

'St Vincent's Private Hospital Sydney, Victoria Street, 2010, Darlinghurst, NSW,

Australia

${ }^{2}$ National Centre for Clinical Outcomes Research, Australian Catholic

University, Sydney, Australia

Full list of author information is available at the end of the article
} 


\section{Background}

Venous thromboembolism (VTE) is a common and potentially devastating complication of hospitalisation. Failure to provide appropriate VTE prophylaxis can result in serious adverse outcomes including symptomatic deep vein thrombosis (DVT) or pulmonary embolism (PE), post-thrombotic syndrome, chronic pulmonary hypertension, recurrent VTE, or fatal PE. Each year in the United States there are an estimated one million cases of VTE resulting in approximately 300,000 deaths annually [1]. Together, the combined morbidity and mortality associated with this disease process result in an estimated economic burden to the nation of $\$ 1.5$ billion/year [2].

People who are hospitalised with acute medical illness are particularly at risk of VTE. Without effective prophylaxis $10-20 \%$ of medical patients will develop an objectively diagnosed VTE which, in turn, has the potential to result in fatal PE. Within the acute patient population, fatal PE accounts for $10 \%$ of all deaths making it the single most preventable cause of hospital related mortality [3]. Contrary to common held perceptions, a significant proportion of these deaths occur in the medical patient population. A retrospective evaluation of 6833 autopsies found that $80 \%$ of the fatal PEs occurred in medical (nonsurgical) patients [4].

VTE in hospitalised medical patients is preventable; evidence-based guidelines recommend the use of low molecular weight heparin, low dose unfractionated heparin, or fondaparinux for patients deemed to be at increased risk of VTE [5,6]. Risk factors for VTE in medical patients include active cancer, previous VTE, reduced mobility, known thrombophylic condition, increased age, heart and/or respiratory failure, myocardial infarction, ischaemia stroke, acute infection or rheumatologic condition, obesity, and ongoing hormonal treatment $[5,6]$. A number of tools have been developed and validated to aid in the assessment of VTE risk and help determine the onset, intensity, type, and duration of recommended prophylaxis [7-9].

Despite the widespread availability of evidence-based guidelines on VTE prevention hospitalised medical patients still receive suboptimal prophylaxis [10-13]. One international study, the ENDORSE study, found that only $40 \%$ of at risk medical patients $(n=37,356)$ were receiving the recommended prophylaxis [12,14]. Numerous strategies to improve VTE prevention in hospitalised patients have been studied with varying degrees of success [15-19]. The evidence suggests that active implementation strategies that engage the target population are more effective than passive strategies at changing clinician behaviour and improving prophylaxis rates $[15,17,19]$.

An educational outreach visit (EOV) is an active implementation strategy that entails a structured one-to- one educational visit conducted in the clinical setting of the participant by a trained facilitator [20]. This intervention is also known as university-based educational detailing, academic detailing, and educational visiting [21]. An EOV is based on social marketing theory. It relies on the psychological principles of persuasion to influence clinician behaviour and promote evidence-based practices [22]. A Cochrane systematic review of this implementation strategy concluded that EOVs, alone or in combination with other interventions, are consistently effective at influencing prescribing practices [21]. There have been few studies, however, examining the clinical impact of EOVs on the provision of VTE prophylaxis to medical patients and no previous studies on its acceptability or utility.

\section{Methods}

\section{Objective}

To evaluate the acceptability, utility and clinical impact of an EOV on doctors' provision of pharmacological VTE prophylaxis to hospitalised medical patients.

\section{Target population}

The target population was doctors who regularly admit medical (nonsurgical) patients to the study site. Regular was defined as being in the top two quartiles of admitters which equated to a minimum of 40 admissions per year.

\section{Setting}

The study site is a 270 bed acute care private hospital in Sydney, Australia. It provides services in all major fields of medicine and surgery with the exception of obstetric and paediatric care. The hospital has approximately 20,000 admissions annually, with approximately $30 \%$ admitted for acute medical illness. As is the case in most private hospitals in Australia, patients are cared for by consulting medical officers with minimal support from junior medical staff.

\section{Ethics}

The study received ethics clearance from the St Vincent's Human Research Ethics Committee (File number 11/051).

\section{Intervention}

A vascular medicine physician with expertise in VTE prevention was recruited to the role of EOV facilitator and was responsible for arranging and conducting each visit. The facilitator followed a strict protocol which was collaboratively developed by a multidisciplinary team of healthcare professionals (see Table 1). A Cochrane systematic review [21] and social marketing literature [22-24] informed the development of the protocol. 
Table 1 Educational outreach visit protocol

\section{EOV component}

Planning the visit

Building trust, credibility and likability

Promoting two-sided communication

Delivering key message(s)

Wrapping up

Providing follow-up

\section{Element}

Contact the target population by email, phone, or in person

Negotiate a convenient time and location for the visit

Reconfirm arrangements prior to the visit

Discuss with the research team any recruitment difficulties

Ensure appropriate space for the discussion

Engage in small talk to place the participant at ease

Explain the purpose of the visit

Negotiate the session length (approximately 20 minutes)

Introduce the four key messages and identify participants specific needs

Mention the key opinion leaders in support of the project

List the project's academic and clinical affiliations

Highlight your own clinical expertise in the area

Attempt to uncover personal similarities between participant and yourself

Offer genuine praise where appropriate

Ask open ended questions

Use minimal encouragement techniques

Paraphrase and reflect on the participants comments

Anticipate and acknowledge controversial issues

Overcome any objections and handle challenging responses

VTE is an important healthcare issue

Assess individual patient risk

Provide evidence-based VTE prophylaxis and patient education

Monitor and reassess each patient during their hospital stay

Reflect on the discussion

Reiterate the key message(s) discussed

Give the participant the printed resource material to keep

Gain commitment to provide evidence-based prophylaxis

Follow-up via email, phone, or in person

Fulfil any commitments made during the visit

events. A concise graphic educational resource was developed to accompany and reinforce the verbal message.

Two trial visits were conducted prior to the intervention period to identify potential issues and familiarise the facilitator with the protocol.

\section{Outcome measures and data collection Acceptability}

Acceptability was measured with post intervention participant and facilitator surveys. The participants' survey and self-addressed envelope were given to the participants by the facilitator following the EOV. The survey contained eight questions in total; six questions related to the doctor's beliefs about the effectiveness of the EOV at increasing knowledge and addressing concerns about VTE prophylaxis for medical patients. The remaining two questions asked participants how likely it was that 
they would participate in a program such as this in the future, and how likely it was that the intervention would influence their clinical practice. The EOV facilitator was also asked to complete a post intervention survey appraising the participants' level of interest, participation and comprehension. All survey questions were answered on a five point likert scale.

\section{Utility}

Descriptive data on the practical application and utility of the intervention were recorded on a data collection form by the EOV facilitator. The information included the time and effort spent arranging the EOV, the time spent conducting the EOV, the number of interruptions and the time spent on them, the location of the EOV, the facilitator's self-assessed adherence to the elements of the study protocol, and whether or not the participant committed to provide evidence-based prophylaxis.

\section{Clinical impact}

Clinical impact was assessed by auditing the proportion of medical patients receiving appropriate pharmacological VTE prophylaxis before and after the EOV intervention. The audits were conducted using an audit tool based on national VTE prevention guidelines [6]. A registered nurse trained in the use of the tool conducted each audit with expert adjudication from a consultant vascular physician when required. VTE prophylaxis was deemed appropriate if it conformed to the locally endorsed guideline with consideration given to individual risk status and contraindications. As per the guideline, patients were classified as high-risk based on the presence of one or more known risk factor (see Table 2). Contraindications to pharmacological prophylaxis included active bleeding; high risk of bleeding; severe hepatic disease; heparin induced thrombocytopenia; or current anticoagulation. The definition of appropriate includes prophylaxis prescribed when indicated; and prophylaxis not prescribed when contraindicated. The following exclusion criteria were used for patient selection: Planned or prior (previous 30 days) surgery on that admission; admitted for less than 24 hours; and inadequate documentation to complete a risk assessment.

\section{Sample size}

The study was designed to detect a change in prescribing practice of $15 \%$ (from $50 \%$ to $65 \%$ appropriate prophylaxis). This estimate of effect was based on two previous studies which had used EOVs to improve VTE prophylaxis in the acute care setting [25,26]. A total sample size of 300 patients ( 150 pre and 150 post intervention) was necessary to power the study at $80 \%$ with a significance level of $5 \%$.

\section{Data analysis}

Data were entered into SPSS version 18 for analysis. Categorical data were summarised as number and percentage and contiguous data were summarised as median and interquartile (IQ) range. For comparisons between groups, the $\mathrm{T}$ test, or Mann-Whitney $\mathrm{U}$ test, was used for continuous variables (age, number of years post registration) and the Chi-square test was used for dichotomous variables (appropriate prophylaxis, risk factors, sex, specialty unit, admitting specialty). The difference in pharmacological prophylaxis rates before and after the intervention was calculated with $95 \%$ confidence intervals. The $\mathrm{p}$ value for statistical significance was set at $<0.05$.

\section{Results}

\section{Characteristics of the target population}

Of the 26 doctors who met the inclusion criteria 19 (73\%) agreed to participate in the intervention and seven (27\%) declined or were unavailable. The demographic characteristics of the target population are shown in Table 3. The median age of the participants was 54 years (IQ range 42-65) and their median number of years post registration was 30 years (IQ range 18-41). Fifteen (79\%) were male and four (21\%) female. The clinical specialties of the doctors were cardiology $(n=8,42 \%)$; neurology $(n=4,21 \%)$; nephrology $(n=1,5.3 \%)$; medical oncology $(n=1,5.3 \%) ;$ immunology/rheumatology $(n=$ $2,10 \%)$; thoracic medicine $(\mathrm{n}=2,10 \%)$; and gastroenterology $(n=1,5.3 \%)$. There was no statistical difference in sex, number of years post registration, or specialty between doctors who received the intervention and those who declined or were unavailable.

\section{Characteristics of the audited patients}

A total of 300 consecutive patients who met the inclusion criteria were audited before $(\mathrm{n}=150)$ and after $(\mathrm{n}=$ 150) the two month EOV intervention period. The demographic characteristics of the audited patients are summarised in Table 4. There were no statistical differences between the two groups in age, sex, admitting specialty, or risk profile. The mean age of the groups was 70.8 (SD 14.4) and 72.4 (SD 13.9) years respectively. The majority of patients in both groups were admitted by a cardiologist $(n=91,60 \%$ and $n=90,60 \%)$. There was no significant difference in risk profile or contraindications to prophylaxis between the two patient groups (Table 2). The majority of patients were identified as being at a high risk of VTE ( $84 \%$ in the pre intervention group and $77 \%$ in the post intervention group).

\section{Acceptability}

Table 5 depicts the results of the participant and facilitator post intervention survey. Sixteen (94\%) of the 17 
Table 2 VTE risk factors \& contraindications to prophylaxis

\begin{tabular}{|c|c|c|c|}
\hline Risk factors \& contraindications & Pre intervention $(n=150)$ & Post intervention $(n=150)$ & $\mathrm{P}$ Value \\
\hline High-risk of VTE & $126(84)$ & $116(77)$ & $0.14 \wedge$ \\
\hline \multicolumn{4}{|l|}{ Risk factors } \\
\hline Ischaemic stroke & $7(4.7)$ & $3(2)$ & $0.19 \wedge$ \\
\hline History of VTE & $15(10)$ & $18(12)$ & $0.58 \wedge$ \\
\hline Active cancer & $4(2.7)$ & $4(2.7)$ & $1.0 \wedge$ \\
\hline Decompensated heart failure & $42(28)$ & $29(19)$ & $0.7 \wedge$ \\
\hline Acute on chronic lung disease & $10(6.7)$ & $10(6.7)$ & $1.0 \wedge$ \\
\hline Age $>60$ years and immobile & $107(71)$ & $108(72)$ & $0.89 \wedge$ \\
\hline Acute inflammatory disease & $6(4)$ & $1(0.7)$ & $0.5 \wedge$ \\
\hline Multiple additional risk factors & $21(14)$ & $10(6.7)$ & $0.33 \wedge$ \\
\hline \multicolumn{4}{|l|}{ Additional risk factors } \\
\hline Immobility (<60 years) & $1(0.7)$ & $1(0.7)$ & $0.98 \wedge$ \\
\hline Familial history of VTE & $1(0.7)$ & $0(0)$ & $0.31 \wedge$ \\
\hline Oestrogen therapy & $2(1.4)$ & $1(0.7)$ & $0.55^{\wedge}$ \\
\hline Obesity & $10(6.8)$ & $7(4.7)$ & $0.43 \wedge$ \\
\hline Thrombophilia & $1(0.7)$ & $0(0)$ & $0.313^{\wedge}$ \\
\hline Active inflammation & $6(4.1)$ & $2(1.4)$ & $0.09 \wedge$ \\
\hline Contraindications & $48(32)$ & $49(32.7)$ & $0.15^{\wedge}$ \\
\hline Active bleeding & $5(3.3)$ & $5(3.3)$ & $1.0 \wedge$ \\
\hline High risk of bleeding & $5(3.3)$ & $7(4.7)$ & $0.55^{\wedge}$ \\
\hline Severe hepatic disease & $1(0.7)$ & 0 & $0.31 \wedge$ \\
\hline Heparin induced thrombocytopenia & $0(0)$ & $0(0)$ & \\
\hline Current anticoagulation & $41(27.3)$ & $38(25.3)$ & $0.69 \wedge$ \\
\hline Other contraindication & $0(0)$ & $2(1.3)$ & $0.15^{\wedge}$ \\
\hline
\end{tabular}

Percentages may not add up to 100 due to missing data. SD Standard Deviation; ^Chi-square.

Table 3 Characteristics of the target population

\begin{tabular}{|c|c|c|c|c|}
\hline \multicolumn{2}{|c|}{ Characteristics } & \multirow{2}{*}{$\begin{array}{l}\text { Received the intervention }(\mathbf{n}=\mathbf{1 9}) \\
54(42-65)\end{array}$} & \multirow{2}{*}{$\begin{array}{l}\text { Declined or unavailable }(n=7) \\
\text { N/A }\end{array}$} & \multirow[t]{2}{*}{$P$ value } \\
\hline Age & Median (IQ range) & & & \\
\hline \multicolumn{2}{|c|}{ Years post registration } & $30(18-41)$ & $26(23-33)$ & $0.93^{*}$ \\
\hline \multirow[t]{3}{*}{ Sex } & Number (\%) & & & $0.18 \wedge$ \\
\hline & & $15(79)$ & $7(100)$ & \\
\hline & & $4(21)$ & $0(0)$ & \\
\hline \multicolumn{2}{|c|}{ Specialty } & & & $0.32^{\wedge}$ \\
\hline \multicolumn{2}{|c|}{ Cardiology } & $8(42)$ & $3(43)$ & \\
\hline \multicolumn{2}{|c|}{ Neurology } & $4(21)$ & $1(14)$ & \\
\hline \multicolumn{2}{|c|}{ Nephrology } & $1(5.3)$ & $0(0)$ & \\
\hline \multicolumn{2}{|c|}{ Medical oncology } & $1(5.3)$ & $0(0)$ & \\
\hline \multicolumn{2}{|c|}{ Immunology/ rheumatology } & $2(10)$ & $0(0)$ & \\
\hline \multicolumn{2}{|c|}{ Thoracic medicine } & $2(10)$ & $0(0)$ & \\
\hline \multicolumn{2}{|c|}{ Gastroenterology } & $1(5.3)$ & $3(43)$ & \\
\hline
\end{tabular}

Percentages may not add up to 100 due to missing data. IQ Interquartile range; N/A not available; ${ }^{*}$ Mann-Whitney U test; $\wedge$ Chi-square. 
Table 4 Characteristics of the audited patients

\begin{tabular}{|c|c|c|c|c|}
\hline \multicolumn{2}{|c|}{ Demographic } & \multirow{2}{*}{$\begin{array}{l}\text { Pre intervention }(\mathbf{n}=\mathbf{1 5 0}) \\
70.8(14.4)\end{array}$} & \multirow{2}{*}{$\begin{array}{l}\text { Post intervention }(\mathbf{n}=\mathbf{1 5 0}) \\
72.4(13.9)\end{array}$} & \multirow{2}{*}{$\frac{\text { P Value }}{0.33^{*}}$} \\
\hline Age & Mean (SD) & & & \\
\hline Sex & Number (\%) & & & $0.9 \wedge$ \\
\hline \multicolumn{2}{|c|}{ Male } & $84(56)$ & $83(55.3)$ & \\
\hline \multicolumn{2}{|c|}{ Female } & $66(44)$ & $67(44.7)$ & \\
\hline \multicolumn{3}{|c|}{ Admitting specialty } & & $0.98 \wedge$ \\
\hline \multicolumn{2}{|c|}{ Cardiology } & $91(61)$ & $90(60)$ & \\
\hline \multicolumn{2}{|c|}{ Oncology } & $3(2)$ & $3(2)$ & \\
\hline \multicolumn{2}{|c|}{ Thoracic medicine } & $6(4)$ & $5(3.3)$ & \\
\hline \multicolumn{2}{|c|}{ Gastroenterology } & $11(7.3)$ & $8(5.3)$ & \\
\hline \multicolumn{2}{|c|}{ Nephrology } & $9(6)$ & $9(6)$ & \\
\hline \multicolumn{2}{|c|}{ Neurology } & $13(8.7)$ & $12(8)$ & \\
\hline \multicolumn{2}{|c|}{ Rheumatology } & $1(0.3)$ & $1(0.3)$ & \\
\hline \multicolumn{2}{|c|}{ Cardiac investigations } & $12(8)$ & $18(12)$ & \\
\hline \multicolumn{2}{|c|}{ Immunology } & $4(2.7)$ & $4(2.7)$ & \\
\hline
\end{tabular}

Percentages may not add up to 100 due to missing data. SD Standard Deviation; ${ }^{* T}$ test; $\wedge$ Chi-square.

participants who returned the post intervention survey reported that the EOV was effective or extremely effective at increasing their knowledge and 15 (88\%) felt that it was effective or extremely effective at addressing their concerns about VTE prophylaxis for medical patients. The participants also agreed that the EOV was effective at providing information on the four key messages outlined in the study protocol: 16 (94\%) participants reported that the EOV was effective or extremely effective at communicating the significance of VTE and the importance of VTE risk assessment; 15 (88\%) agreed that the EOV was effective or extremely effective at providing information on selecting appropriate VTE prophylaxis; and 10 (59\%) felt that the EOV was effective or extremely effective at providing information about ongoing monitoring. When asked how likely it was that

Table 5 Acceptability of the Educational Outreach Visit

\begin{tabular}{|c|c|c|c|c|c|}
\hline How effective was the Educational Outreach Visit in... & $\begin{array}{c}\text { Extremely } \\
\text { ineffective } \\
n(\%)\end{array}$ & $\begin{array}{c}\text { Ineffective } \\
\text { n (\%) }\end{array}$ & $\begin{array}{l}\text { Unsure } \\
\text { n (\%) }\end{array}$ & $\begin{array}{c}\text { Effective } \\
\text { n (\%) }\end{array}$ & $\begin{array}{c}\text { Extremely } \\
\text { effective } \\
n(\%)\end{array}$ \\
\hline $\begin{array}{l}\text { Increasing or refreshing your knowledge about VTE prophylaxis } \\
\text { for medical patients? }\end{array}$ & $0(0)$ & $1(5.3)$ & $0(0)$ & $11(58)$ & $5(26)$ \\
\hline $\begin{array}{l}\text { Addressing concerns you have had about providing VTE prophylaxis } \\
\text { to medical patients? }\end{array}$ & $0(0)$ & $1(5.3)$ & $0(0)$ & $13(68)$ & $2(11)$ \\
\hline Providing information about the significance of VTE as a healthcare issue? & $0(0)$ & $1(5.3)$ & $0(0)$ & $11(58)$ & $5(26)$ \\
\hline Providing information about VTE risk assessment for medical patients? & $0(0)$ & $1(5.3)$ & $0(0)$ & $11(58)$ & $5(26)$ \\
\hline $\begin{array}{l}\text { Providing information about selecting appropriate VTE prophylaxis for } \\
\text { medical patients? }\end{array}$ & $0(0)$ & $1(5.3)$ & $1(5.3)$ & $11(58)$ & $4(21)$ \\
\hline $\begin{array}{l}\text { Providing information about the ongoing monitoring of patients risk } \\
\text { and response to prophylaxis? }\end{array}$ & $0(0)$ & $3(16)$ & $4(21)$ & $7(37)$ & $3(16)$ \\
\hline How likely is it that... & Extremely unlikely & Unlikely & Unsure & Likely & Extremely likely \\
\hline $\begin{array}{l}\text { You will participate in another educational program such as this one } \\
\text { in the future? }\end{array}$ & $1(5.3)$ & $0(0)$ & $3(16)$ & $11(58)$ & $2(11)$ \\
\hline This educational visit will influence your clinical practice? & $1(5.3)$ & $0(0)$ & $3(16)$ & $11(58)$ & $2(11)$ \\
\hline What was the participants perceived level of... & Very low & Low & Average & High & Very high \\
\hline Interest in the topic presented? & $2(11)$ & $3(16)$ & $3(16)$ & $6(32)$ & $5(26)$ \\
\hline Participation during the visit? & $1(5.3)$ & $1(5.3)$ & $4(21)$ & $3(16)$ & $10(53)$ \\
\hline Comprehension of the information provided? & $0(0)$ & $0(0)$ & $2(11)$ & $7(37)$ & $10(53)$ \\
\hline
\end{tabular}

Percentages may not add up to 100 due to missing data. 
they would participate in another EOV, 12 (71\%) participants reported that it would be likely, or extremely likely. The same number $(n=12,71 \%)$ felt that the EOV was likely, or extremely likely to influence their clinical practice. When the EOV facilitator was asked to rate the participants' $(\mathrm{n}=19)$ perceived interest, participation and comprehension in the EOV he reported that 11 (58\%) participants had a high or very high level of interest; 13 (68\%) had a high or very high level of participation; and 17 (89\%) had a high or very high level of comprehension.

\section{Utility}

Table 6 shows the descriptive data on the practical application and utility of the intervention. The median number of times it was necessary to make contact with participants to arrange the EOV was 3 (IQ range 1 to 4 ). The median time spent on each EOV was 92 minutes (IQ range 78 to 129) which was made up of time spent arranging the EOV (median 10 minutes, IQ range 10 to 20); customising the material (median 45 minutes, IQ range 45 to 60); waiting for the participant (median 5 minutes, IQ range 0-20) and conducting the EOV (median 15 minutes, IQ range 15 to 20 ). The majority of visits were conducted in the doctor's office $(n=10,53 \%)$. The remainder were held in the clinical area $(n=6,32 \%)$; other public area $(\mathrm{n}=2,10 \%)$; or other private area $(\mathrm{n}=1,5 \%)$. At the completion of the EOV 15 (78\%) of the 19 participants gave a verbal commitment to provide evidence-based prophylaxis. The facilitator's self-reported adherence to all of the elements of the EOV protocol was $80 \%$ (IQ range $70-85$ ).

\section{Clinical impact}

There was a significant improvement in the proportion of medical patients who received appropriate pharmacological VTE prophylaxis following the intervention (54\% to $70 \%$, $16 \%$ improvement, 95\% CI 5 to $26, \mathrm{p}=0.004$ ). Removing patients who were at lower risk of VTE from the analysis made no difference to the significance of the result (47\% to $63 \%, 16 \%$ improvement, $95 \%$ CI 3 to $27, \mathrm{p}=0.01$ ).

\section{Discussion}

VTE is a major health and financial burden on the community [3]. Unfortunately, despite the availability of evidence-based guidelines, VTE prophylaxis is still frequently underutilised. Our study found that at baseline only $54 \%$ of medical patients were receiving evidencebased VTE prophylaxis. This confirms the evidencepractice gap described in the international literature [10-13]. Numerous strategies to improve VTE prevention in hospitalised patients have been studied but none have been successful at addressing all the barriers to the provision of evidence-based care [15-19].

The barriers to the provision of appropriate medical patient prophylaxis have been documented in a number $[27,28]$ of recent studies. Known barriers include a lack

Table 6 Utility of the educational outreach visit

\begin{tabular}{|c|c|}
\hline Measures & Median (IQ range) \\
\hline \multicolumn{2}{|l|}{ Number of contacts needed to arrange the EOV } \\
\hline Number of contacts needed to arrange the visit & $3(1-4)$ \\
\hline Number of cancelled visits prior to the visit & 0 \\
\hline \multicolumn{2}{|l|}{ Time spent arranging and conducting the EOV ( $\mathrm{min})$} \\
\hline Time spent arranging the visit & $20(10-20)$ \\
\hline Time spent customising material & $45(45-60)$ \\
\hline Time spent waiting for the participant & $5(0-20)$ \\
\hline Time spent with the participant during the visit & $15(15-20)$ \\
\hline Time spent on interruptions & 0 \\
\hline Total time spent on the visit & $92(78-129)$ \\
\hline \multicolumn{2}{|l|}{ Protocol adherence } \\
\hline Percent of protocol elements delivered to participant & $80(70-85)$ \\
\hline Location of the EOV & Number (\%) \\
\hline Clinical area & $6(32)$ \\
\hline Office & $10(53)$ \\
\hline Other public area & $2(10)$ \\
\hline Other private area & $1(5)$ \\
\hline \multicolumn{2}{|l|}{ Outcome of the EOV } \\
\hline Participant agreed to provide evidence-based prophylaxis & $15(79)$ \\
\hline
\end{tabular}


of awareness of the importance of VTE prophylaxis and of the presence of evidence-based guidelines; a lack of knowledge on the indications for VTE prophylaxis and on appropriate prophylaxis options; and a lack of agreement and acceptance of current evidence-based recommendations [27,28]. EOVs acknowledge and address each participant's barriers to change with the aim of facilitating increased compliance with evidence-based practice [20]. Few studies have examined the clinical impact of this intervention on the provision of VTE prophylaxis to medical patients and no previous studies have reported on its acceptability or utility.

Our results strongly suggest that EOVs are an acceptable implementation strategy for doctors working in the acute care setting. Nineteen (73\%) of the 26 doctors eligible to participate agreed to receive an EOV. This was a greater than expected uptake given the established difficulty in providing hospital delivered education to senior doctors who, in the Australian private system, are consultant practitioners and not employees of the hospital [29]. It was also encouraging to find that following the intervention $71 \%(\mathrm{n}=12)$ of participants who provided feedback reported that they would participate in another EOV in the future.

By reporting descriptive data on the practical application and utility of the intervention we hope to provide valuable information for anyone wishing to use this intervention in an acute care hospital setting. Of particular note was the considerable time (92 minutes) required to organise, prepare and deliver each EOV. This study is one of a very few published studies to report the total time required for each EOV and the only study set in an acute care hospital setting.

Of the 19 participants who received the intervention $79 \%(\mathrm{n}=15)$ gave a verbal agreement to provide evidence-based VTE prophylaxis to their medical patients. Importantly, this commitment translated into a $16 \%$ ( $95 \%$ CI 5 to $26, \mathrm{p}=0.004)$ improvement in prophylaxis rates above baseline. This clinical impact is much larger than that reported in a Cochrane systematic review on the effectiveness of EOVs [21]. The review found that the median adjusted risk difference in compliance with prescribing practices was only $4.8 \%$ (IQ range $3.0 \%$ to $6.5 \%$ ). The findings are similar, however, to two previous studies which used EOVs to improve doctors' compliance with evidence-based VTE prevention practices in the acute care hospital setting. Roberts and Adams [25] observed a $14.2 \%$ (52.8\% to $67 \%, \mathrm{p}=$ 0.004 ) improvement in prophylaxis rates in medical patients while Grupper et al. [26] reported a $21 \%$ (29\% to $50 \%, \mathrm{p}<0.001)$ improvement in a surgical population.

A limitation of our study was the use of a beforeand-after design which may be subject to methodological limitations. There is some evidence to suggest that uncontrolled before and after studies over-estimate the effect of interventions [30]. Having only one postimplementation data point collected three months after the intervention means that we cannot know if the observed improvement in practice will be sustained or improved upon over time. The hospital plans to monitor the sustainability of the improvement as part of the hospitals ongoing quality systems. Future research is recommended that examines the clinical impact of EOVs on VTE prophylaxis using a cluster randomised controlled trial which includes an evaluation of the ongoing sustainability of the intervention.

\section{Conclusion}

This study confirms that EOVs are effective at improving doctors' provision of pharmacological VTE prophylaxis to hospitalised medical patients. In addition, it provides evidence of the acceptability of the intervention as an implementation strategy in the acute care setting, as well as valuable data on the practical application and utility of EOVs for those wishing to use this intervention in the future.

\section{Abbreviations}

VTE: Venous thromboembolism; PE: Pulmonary embolism; DVT: Deep vein thrombosis; EOV: Educational outreach visit.

\section{Competing interests}

The authors declare that they have no competing interests.

\section{Authors' contributions}

All authors contributed to the study concept, design and preparation of the manuscript. JD, AO and KW developed the intervention protocol. JD collected and analysed the data. All authors read and approved the final manuscript.

\section{Acknowledgements}

The authors wish to acknowledge the financial support of the St Vincent Clinic Foundation and thank them for their ongoing patronage of multidisciplinary research on the St Vincent's campus. The St Vincent's Clinic Foundation had no role in the design; in the collection, analysis, and interpretation of data; in the writing of the manuscript; or the decision to submit the manuscript for publication.

\section{Author details}

${ }^{1}$ St Vincent's Private Hospital Sydney, Victoria Street, 2010, Darlinghurst, NSW, Australia. ${ }^{2}$ National Centre for Clinical Outcomes Research, Australian Catholic University, Sydney, Australia. ${ }^{3}$ University of Tasmania School of Nursing \& Midwifery, Education Centre, 1 Leichhardt St, 2010, Darlinghurst, NSW, Australia. ${ }^{4}$ Nursing Research Institute, St Vincent's \& Mater Health SydneyAustralian Catholic University, St Vincent's Hospital, Victoria Street, 2010, Darlinghurst, NSW, Australia.

Received: 14 November 2012 Accepted: 25 September 2013 Published: 8 October 2013

\section{References}

1. Heit JA, O'Fallon WM, Petterson TM, Lohse CM, Silverstein MD, Mohr DN, Melton $\sqcup$ III: Relative impact of risk factors for deep vein thrombosis and pulmonary embolism: A population-based study. Arch Intern Med 2002, 162(11):1245.

2. Dobesh PP: Economic burden of venous thromboembolism in hospitalized patients. Pharmacotherapy 2009, 29(8):943-953. 
3. Economics A: The burden of venous thromboembolism in Australia, report for the Australia and New Zealand working party on the management and prevention of venous thromboembolism. Sydney: Access Economics; 2008.

4. Alikhan $\mathrm{R}$, Peters F, Wilmott $\mathrm{R}$, Cohen A: Fatal pulmonary embolism in hospitalised patients: a necropsy review. J Clin Pathol 2004, 57(12):1254.

5. Kahn SR, Lim W, Dunn AS, Cushman M, Dentali F, Akl EA, Cook DJ, Balekian $A A$, Klein RC, Le H, et al: Prevention of VTE in nonsurgical patients: American college of chest physicians evidence-based clinical practice guidelines. Chest 2012, 141(2_suppl):e195S-e226S.

6. The Australian and New Zealand Working Party on the Management and Prevention of Venous Thromboembolism: Prevention of venous thromboembolism: best practice guidelines for Australia and New Zealand (5th ed). Sydney: Health Education and Management Innovations; 2010.

7. Caprini J, Hyers T: Compliance with antithrombotic guidelines. Manag Care 2006, 15(9):49-50.

8. Cohen A, Alikhan $\mathrm{R}$, Arcelus J: A risk assessment model for identifying medical patients who should receive thromboprophylaxis. J Thromb Haemost 2003, 1(Supp):OC437.

9. Kucher N, Koo S, Quiroz R: Electronic alerts to prevent venous thromboembolism among hospitalized patients. J Vasc Surg 2005, 42(1):178.

10. Amin A, Spyropoulos A, Dobesh P, Shorr A, Hussein M, Mozaffari E, Benner J: Are hospitals delivering appropriate VTE prevention? the venous thromboembolism study to assess the rate of thromboprophylaxis (VTE start). J Thromb Thrombolysis 2010, 29(3):326-339.

11. Rothberg MB, Lahti M, Pekow PS, Lindenauer PK: Venous thromboembolism prophylaxis among medical patients at US hospitals. J Gen Intern Med 2010, 25(6):489-494.

12. Bergmann JF, Cohen AT, Tapson VF, Goldhaber SZ, Kakkar AK, Deslandes B, Huang W, Anderson FA: Venous thromboembolism risk and prophylaxis in hospitalised medically ill patients. The ENDORSE global survey. Throbosis and Haemostasis 2010, 103(4):736-748.

13. Tapson VF, Decousus H, Pini M, Chong BH, Froehlich JB, Monreal M, Spyropoulos AC, Merli GJ, Zotz RB, Bergmann JF: Venous thromboembolism prophylaxis in acutely III hospitalized medical patients. Chest 2007, 132(3):936-945.

14. Geerts W, Bergqvist D, Pineo G, Heit J, Samama C, Lassen M, Colwell C, P ACoC: Prevention of venous thromboembolism: American college of chest physicians evidence-based clinical practice guidelines (8th edition). Chest 2008, 133(6 Suppl):381S-453S.

15. Tooher R, Middleton P, Pham C, Fitridge R, Rowe S, Babidge W, Maddern G: A systematic review of strategies to improve prophylaxis for venous thromboembolism in hospitals. Ann Surg 2005, 241(3):397-415.

16. Amin AN, Deitelzweig SB: Optimizing the prevention of venous thromboembolism: recent quality initiatives and strategies to drive improvement. Joint Comm J Qual Patient Saf 2009, 35(11):558-564.

17. Merli G: Improving venous thromboembolism performance: a comprehensive guide for physicians and hospitalists. Hosp Pract 2010, 38(3):7-16.

18. Kakkar A, Davidson B, Haas S: Compliance with recommended prophylaxis for venous thromboembolism: Improving the use and rate of uptake of clinical practice guidelines. J Thromb Haemost 2004, 2(2):221-227.

19. Michota FA: Bridging the gap between evidence and practice in venous thromboembolism prophylaxis: The quality improvement process. J Gen Intern Med 2007, 22(12):1762-1770.

20. Soumerai $\mathrm{S}$, Avorn J: Principles of educational outreach ('academic detailing') to improve clinical decision making. JAMA 1990, 263(4):549-556.

21. O'Brien M, Rogers $S$, Jamtvedt G, Oxman A, Odgaard-Jensen J, Kristoffersen D, Forsetlund L, Bainbridge D, Freemantle N, Davis D: Educational outreach visits: effects on professional practice and health care outcomes. Cochrane Database Syst Rev 2007, 4:1-79.

22. Morris ZS, Clarkson PJ: Does social marketing provide a framework for changing healthcare practice? Health Policy 2009, 91(2):135-141.

23. Opel DJ, Diekema DS, Lee NR, Marcuse EK: Social marketing as a strategy to increase immunization rates. Arch Pediatr Adolesc Med 2009, 163(5):432-437.

24. Cialdini RB: Harnessing the science of persuasion. (Cover story). Harv Bus Rev 2001, 79(9):72-79.

25. Roberts G, Adams R: Impact of introducing anticoagulation-related prescribing guidelines in a hospital setting using academic detailing. Ther Clin Risk Manag 2006, 2(3):309-316.
26. Grupper A, Rudin D, Drenger B, Varon D, Gilon D, Gielchinsky Y, Menashe M, Mintz $Y$, Rivkind A, Brezis M: Prevention of perioperative venous thromboembolism and coronary events: Differential responsiveness to an intervention program to improve guidelines adherence. International J Qual Health Care 2006, 18(2):123-126.

27. Vardi M, Dagna L, Haran M, Duckit R: Attitudes towards and practice of venous thromboembolism prevention in general internal medicine wards: a multinational survey from member countries of the European federation of internal medicine. Thrombosis research 2011, 129(5):573-576.

28. Lloyd NS, Douketis JD, Cheng J, Schünemann HJ, Cook DJ, Thabane L, Pai M, Spencer FA, Haynes RB: Barriers and potential solutions toward optimal prophylaxis against deep vein thrombosis for hospitalized medical patients: a survey of healthcare professionals. J Hosp Med 2012, 7(1):28-34

29. Koczwara B, Barton MB, Tattersall MH, Turner DR, Olver IN, Starmer DL: Medical education in Australia: changes are needed. Med J Aust 2006, 3(185):1.

30. Grimshaw J, Campbell M, Eccles M, Steen N: Experimental and quasiexperimental designs for evaluating guideline implementation strategies. Fam Pract 2000, 17(90001):11-16.

\section{doi:10.1186/1472-6963-13-398}

Cite this article as: Duff et al:: Educational outreach visits to improve venous thromboembolism prevention in hospitalised medical patients: a prospective before-and-after intervention study. BMC Health Services Research 2013 13:398.

\section{Submit your next manuscript to BioMed Central and take full advantage of:}

- Convenient online submission

- Thorough peer review

- No space constraints or color figure charges

- Immediate publication on acceptance

- Inclusion in PubMed, CAS, Scopus and Google Scholar

- Research which is freely available for redistribution

Submit your manuscript at www.biomedcentral.com/submit
C BioMed Central 